\title{
Factores asociados al retraso de estudios y de graduación en estudiantes de maestría y doctorado de la Región Cusco, 2013
}

\author{
Doctora Cirujana Dentista Mary Ángela Sosa Huamán \\ (Docente de la Escuela Profesional de Estomatología, Cirujana Dentista, Doctora en Educación)
}

\section{RESUMEN}

El trabajo de investigación titulado «Factores asociados al retraso de estudios y de graduación en estudiantes de maestría y doctorado en universidades de Cusco, 2013», se desarrolló con el aporte de múltiples elementos que coadyuvaron para alcanzar los objetivos planteados.

Para su ejecución, se usaron métodos y materiales necesarios y adecuados de tipo transversal, muestreo probabilístico (214 estudiantes), que cumplieron con los criterios de selección. Se aplicó la técnica del cuestionario para el estudio, donde se realizaron preguntas sobre factores institucionales, personales que influyeron en el retraso de estudios y graduación. Se realizó el análisis estadístico univariado y bivariado.

El retraso de estudios en estudiantes de maestría se presenta, mayoritariamente, durante el 2.do y 3.er ciclos de estudios. Mientras que para el nivel de doctorado no se presentó retraso de estudios. El retraso en la graduación de estudiantes se manifestó, en su mayoría, durante la fase de proyecto de investigación, tanto para maestría como para doctorado.

En cuanto a los factores asociados al retraso de estudios de maestría, los factores institucionales y los factores personales fueron determinantes, mientras que para el doctorado, no se presentó dilación de estudios. Para el retraso en la graduación de los estudiantes de maestría y doctorado, los factores personales y los factores institucionales fueron determinantes para esta postergación: la motivación, el aspecto laboral, el aspecto económico, la coordinación administrativa y la realización de la tesis fueron concluyentes.

La hipótesis fue parcialmente comprobada, puesto que, no solo los factores personales se asociaron al retraso de estudios y graduación, también lo fueron los factores institucionales. 
Culminada la etapa investigadora llegamos a la conclusión: los factores personales y los institucionales fueron determinantes para el retraso de estudios y la graduación de estudiantes en maestría y doctorado en las universidades de Cusco, 2013.

PALABRAS CLAVE: Factores institucionales, personales, sociales, retraso de estudios, retraso de graduación, posgrado. 


\section{ABSTRACT}

The research work entitled "Factors associated to the delay of studies and graduation in masters and doctoral students at universities in Cusco, 2013", was developed with the contribution of multiple elements which contributed to achieve the objectives. For its implementation, were used methods and materials needed and appropriate crosssectional type, probability sampling (214 students), which met the selection criteria. The technique was used in the questionnaire for the study, where questions were asked about institutional factors, personal that influenced the delay of studies and graduation. The statistical analysis was carried out univariate and bivariate.

The delay of studies in Masters students occurs mostly during the 2nd and 3er cycles of studies. While for the doctoral level was not presented delay of studies. The delay in the graduation of students was expressed, in its majority, during the phase of research project, both for mastery as for doctorate.

With regard to the factors associated with the delay of master studies, institutional factors and the personal factors were decisive, whereas for the doctorate, was not presented delay of studies. For the delay in the graduation of the masters and doctoral students, the personal factors and institutional factors were decisive for this postponement: motivation, the labor side, the economic aspect, the administrative coordination and the realization of the thesis were conclusive.

The hypothesis was partially checked, since not only the personal factors were associated to the delay of studies and graduation, so too were the institutional factors. Upon completion of the stage researcher arrived at the conclusion: the personal factors and institutional were decisive for the delay of studies and graduation of students in masters and doctorate programs in the universities of Cusco, 2013.

Key words: institutional factors, personal, social delay of studies, delay of graduation, graduate. 


\section{INTRODUCCIÓN}

La educación superior y la de posgrado formal, tiene sus antecedentes en la creación de la universidad en Alemania, en 1808, cuando se la concibe como un centro formador de profesionales que contribuye a la creación, aplicación y transmisión de nuevos conocimientos. En el caso de la universidad latinoamericana, esta se organizó con énfasis en la docencia y marcada influencia de la iglesia católica. Hacia mediados del siglo XIX, la universidad admitió en su estructura el modelo francés (facultades, escuelas), cuya función es la preparación de los profesionales requeridos por la sociedad. En el siglo XX, los estudios de posgrado se extendieron a todos los continentes $y$, en la actualidad, se consideran esenciales para el crecimiento humano, constituyéndose en el nivel educativo con mayor producción intelectual. Por lo tanto, el aumento de estos se encuentra estrechamente ligado a las demandas y exigencias de la sociedad.

Es oportuno destacar que, en la actualidad, existe una creciente preocupación entre los educadores por la evaluación de los estudios de posgrado, la cual puede ser considerada como parte de la evaluación institucional y acreditación que requieren las autoridades y los miembros de la comunidad con respecto a los cambios, y de este modo, contribuir al desarrollo de una educación de calidad.

Ante una realidad que demanda la formación profesional de nuevas carreras, con énfasis en la investigación y en el desarrollo de actividades de producción intelectual, el abordaje de los factores asociados al retraso de graduación de los estudios de posgrado es clave para definir e interpretar los indicadores y criterios de calidad académica que identifican a un determinado programa.

La problemática — definida como un porcentaje elevado de estudiantes que abandonan los estudios-, ocasiona elevados costos, tanto para el individuo como para la institución. Los estudiantes obtienen un conocimiento actualizado y significativo, pero a un costo considerable — como la pérdida de autoestima, de tiempo, esfuerzo, así como de dinero-, además de generarse sentimientos de frustración. Por otro lado, la institución y los profesores pierden su tiempo, esfuerzo y recursos invertidos en los estudiantes. 
A nivel mundial algunos autores se han enfocado en la búsqueda y análisis de los factores preponderantes. En este recorrido se destaca el estudio de Garbanzo Vargas (2007), y los clasifica en tres determinantes: sociales, personales e institucionales.

El conocer ¿cuáles son los factores asociados al retraso de estudios y retraso de graduación en estudiantes de maestría y doctorado?, implica saber los factores por los cuales algunos profesionales, al empezar estudios a nivel de posgrado, no planifican el tiempo y el esfuerzo que necesitarán para obtener el respectivo grado académico, así como, ¿cuál sería la responsabilidad de las instituciones que ofrecen este servicio académico? La experiencia personal, experiencias de personas cercanas al entorno $\mathrm{y}$, los datos alarmantes del último censo universitario, son las principales motivaciones para conceptualizar el problema y plantearlo ahora en el presente trabajo de investigación.

\section{MATERIALES Y MÉTODOS}

La investigación se realizó en la Provincia y Región Cusco. Está referido a los meses de marzo a mayo del año 2013. Las unidades es estudio fueron: estudiantes o egresados de maestría y doctorado de universidades en el Cusco. En el nivel de maestría tenemos a 1048 alumnos; en doctorado son 28, haciendo un total de 1076 alumnos.

Por ser un universo mayor a 500 unidades de estudio, se utilizará la opción de muestra, para lo cual se considera una confiabilidad de $95 \%$ y un margen de error de $5 \%$. La muestra fue de 214 estudiantes con retraso de estudios y egresados con retraso de graduación.

Se coordinó con los directores de las escuelas de posgrado de la Región Cusco, con la finalidad de aplicar la cédula de preguntas. Se remarca el carácter anónimo del formulario, así como la sinceridad de las respuestas emitidas para contribuir al éxito del estudio. Es necesario informar a los preguntados para su participación voluntaria. Los recursos utilizados fueron: la investigadora, dos personas seleccionadas y capacitadas para la consignación de los datos en la encuesta, un estadista. Se utilizó la infraestructura de las escuelas de posgrado. El instrumento empleado fue el formulario de preguntas, el mismo que será previamente validado mediante la autorización de expertos. 


\section{RESULTADOS}

De acuerdo con los resultados de la investigación, el retraso de estudios de los estudiantes de maestría está asociado a factores institucionales y factores personales. En el caso del doctorado, el retraso de estudios no es dominante. En cuanto a la postergación de la graduación, los factores institucionales y personales son relevantes tanto para la maestría como para el doctorado. Estos resultados indican claramente que el retardo en los estudios y graduación de los estudiantes, están asociados a factores que dependen, esencialmente, de la institución educativa y del propio estudiante. Entre los factores institucionales se encuentran las deficiencias de coordinación administrativa, seguido de las dificultades propias de la realización de la tesis y las dificultades inherentes a los requisitos de grado. Es decir, la publicación o comunicación oportuna de notas, lecturas, reglamentos, realización de trámites administrativos, así como las dificultades relacionadas al desempeño de los asesores de tesis y las dificultades relacionadas al trámite y cumplimiento de los requisitos de grado de los estudiantes, se encuentran entre las razones institucionales que motivan el retraso en los estudios y graduación de los estudiantes de posgrado. Estos datos evidencian la responsabilidad que tienen las instituciones educativas para la continuidad de estudios y graduación de sus estudiantes, responsabilidad que debe ser asumida y corregida con una mejor organización administrativa y académica.

De manera específica, entre los factores personales asociados al retraso en los estudios y graduación se pueden destacar los de índole motivacional, laboral y económico. Estos tres factores están fuertemente asociados: la situación económica y laboral de los estudiantes es muy sensible a las variaciones y dificultades derivadas de los factores institucionales, habida cuenta, que la mayoría de ellos autofinancia sus estudios de posgrado, lo que significa que buena parte de su tiempo está dedicado al cumplimiento de sus responsabilidades laborales. Dicha sensibilidad puede ser traducida en una desmotivación con respecto a los programas de estudios, si es que estos últimos no satisfacen las expectativas de los estudiantes. De hecho, un porcentaje significativo de estudiantes señala la motivación como el principal factor personal responsable de su retraso en los estudios y posterior graduación, pero, como se ha indicado antes, dicha motivación tiende a constituirse más en un efecto que en una causa, puesto que, de por medio están: la calidad de la enseñanza, la calidad de la organización administrativa, la calidad de la asesoría de tesis, etcétera. 
Factores institucionales y el retraso de estudios en los estudiantes de maestría y doctorado

\begin{tabular}{|c|c|c|c|c|c|c|c|c|c|c|}
\hline \multirow{4}{*}{$\begin{array}{l}\text { Retraso de } \\
\text { estudios }\end{array}$} & \multicolumn{8}{|c|}{ FACTORES INSTITUCIONALES } & \multirow{3}{*}{\multicolumn{2}{|c|}{ TOTAL }} \\
\hline & \multicolumn{4}{|c|}{ Maestría } & \multicolumn{4}{|c|}{ Doctorado } & & \\
\hline & \multicolumn{2}{|l|}{ Sí } & \multicolumn{2}{|c|}{ No } & \multicolumn{2}{|c|}{ Sí } & \multicolumn{2}{|c|}{ No } & & \\
\hline & $\mathrm{F}$ & $\%$ & $\mathrm{~F}$ & $\%$ & $\mathrm{~F}$ & $\%$ & $F$ & $\%$ & $F$ & $\%$ \\
\hline 1.er ciclo & 1 & 2 & 4 & 8 & 0 & 0 & 0 & 0 & 5 & 11 \\
\hline 2.do ciclo & 10 & 21 & 5 & 11 & 0 & 0 & 0 & 0 & 15 & 31 \\
\hline 3.er ciclo & 10 & 21 & 5 & 11 & 0 & 0 & 0 & 0 & 15 & 31 \\
\hline 4.to ciclo & 7 & 15 & 5 & 10 & 1 & 2 & 0 & 0 & 13 & 27 \\
\hline Total & 28 & 58 & 19 & 40 & 1 & 2 & 0 & 0 & 48 & 100 \\
\hline
\end{tabular}

Fuente: Elaboración personal

Factores personales y el retraso de estudios en los estudiantes de maestría y doctorado

\begin{tabular}{|c|c|c|c|c|c|c|c|c|c|c|}
\hline \multirow{4}{*}{$\begin{array}{l}\text { Retraso } \\
\text { de } \\
\text { estudios }\end{array}$} & \multicolumn{8}{|c|}{ FACTORES PERSONALES } & \multirow{3}{*}{\multicolumn{2}{|c|}{ TOTAL }} \\
\hline & \multicolumn{4}{|c|}{ Maestría } & \multicolumn{4}{|c|}{ Doctorado } & & \\
\hline & \multicolumn{2}{|l|}{ Sí } & \multicolumn{2}{|c|}{ No } & \multicolumn{2}{|c|}{ Sí } & \multicolumn{2}{|l|}{$\mathrm{Nc}$} & & \\
\hline & $\mathrm{F}$ & & $\mathrm{F}$ & $\%$ & & & $\mathrm{~F}$ & $\%$ & $\mathrm{~F}$ & $\%$ \\
\hline 1.er ciclo & 4 & & 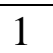 & 7 & & & 0 & 0 & 5 & 11 \\
\hline 2.do c & & & & & & & 0 & 0 & 15 & 31 \\
\hline $\mathrm{rc}$ & 14 & & 2 & 2 & & & 0 & 0 & 15 & 31 \\
\hline 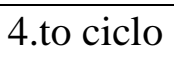 & 10 & & 2 & 4 & & & 0 & 0 & 13 & 27 \\
\hline Tot: & 40 & 8 & 7 & 15 & & 2 & 0 & 0 & 48 & 100 \\
\hline
\end{tabular}

Fuente: Elaboración personal 
Factores institucionales y el retraso de graduación de los estudiantes de maestría y doctorado

\begin{tabular}{|c|c|c|c|c|c|c|c|c|c|c|c|c|c|c|c|}
\hline \multirow{4}{*}{$\begin{array}{l}\text { Retraso de } \\
\text { graduación }\end{array}$} & \multicolumn{13}{|c|}{ FACTORES INSTITUCIONALES } & \multirow{3}{*}{\multicolumn{2}{|c|}{ TOTAL }} \\
\hline & \multicolumn{4}{|c|}{ Maestría } & \multicolumn{4}{|c|}{ Doctorado } & \multicolumn{5}{|c|}{ Ambas } & & \\
\hline & \multicolumn{2}{|l|}{ Sí } & \multicolumn{2}{|c|}{ No } & \multicolumn{2}{|l|}{ Sí } & \multicolumn{2}{|c|}{ No } & & \multicolumn{2}{|c|}{$\overline{\text { Sí }}$} & \multicolumn{2}{|c|}{ No } & & \\
\hline & $\mathrm{F}$ & $\%$ & $\mathrm{~F}$ & $\%$ & $\mathrm{~F}$ & $\%$ & $\mathrm{~F}$ & Th & & & & $\mathrm{F}$ & $\%$ & $\mathrm{~F}$ & $\%$ \\
\hline Sin proyecto & 27 & 16 & 7 & 4 & 9 & 6 & 0 & 0 & 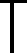 & & .5 & 0 & 0 & 44 & 27 \\
\hline Con proyecto & 60 & 36 & 23 & 14 & 12 & 7 & 1 & 1 & 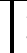 & & & 4 & 2 & 103 & 62 \\
\hline $\begin{array}{ll}\text { Toma } & \text { de } \\
\text { datos } & \end{array}$ & 5 & 3 & 0 & 0 & 0 & 0 & 0 & 0 & & & & 0 & 0 & 5 & 3 \\
\hline Informe & 10 & 6 & 1 & 1 & 2 & 1 & 0 & 0 & & & .5 & 0 & 0 & 14 & 8 \\
\hline Total & 102 & 61 & 31 & 19 & 23 & 14 & 1 & 1 & & & & 4 & 2 & 166 & 100 \\
\hline
\end{tabular}

Fuente: Elaboración personal

Factores personales y retraso de graduación de los estudiantes de maestría y doctorado

\begin{tabular}{|c|c|c|c|c|c|c|c|c|c|c|c|c|c|c|}
\hline \multirow{4}{*}{$\begin{array}{l}\text { Retraso de } \\
\text { graduación }\end{array}$} & \multicolumn{12}{|c|}{ FACTORES PERSONALES } & \multirow{3}{*}{\multicolumn{2}{|c|}{ TOTAL }} \\
\hline & \multicolumn{4}{|c|}{ Maestría } & \multicolumn{4}{|c|}{ Doctorado } & \multicolumn{4}{|c|}{ Ambos } & & \\
\hline & \multicolumn{2}{|l|}{$\mathrm{Si}$} & \multicolumn{2}{|l|}{ No } & \multicolumn{2}{|l|}{$\mathrm{Si}$} & \multicolumn{2}{|c|}{ No } & \multicolumn{2}{|c|}{$\mathrm{Si}$} & \multicolumn{2}{|c|}{ No } & & \\
\hline & $\mathrm{F}$ & $\%$ & $\mathrm{~F}$ & $\%$ & $\mathrm{~F}$ & $\%$ & $\mathrm{~F}$ & $\%$ & $\mathrm{~F}$ & $\%$ & $\mathrm{~F}$ & $\%$ & $\mathrm{~F}$ & $\%$ \\
\hline Sin proyecto & 34 & 21 & 4 & 12 & 10 & 4 & 3 & 2 & 0 & 0 & 0 & 0 & 48 & 29 \\
\hline Con proyecto & 64 & 39 & 14 & 9 & 9 & 5 & 4 & 2 & 4 & 2 & 4 & 2 & 99 & 60 \\
\hline $\begin{array}{ll}\text { Toma } & \mathrm{de} \\
\text { datos }\end{array}$ & 4 & 2 & 1 & 1 & 0 & 0 & 0 & 0 & 0 & 0 & 0 & 0 & 5 & 3 \\
\hline Informe & 10 & 6 & 2 & 1 & 2 & 1 & 0 & 0 & 1 & 1 & 0 & 0 & 14 & 8 \\
\hline Total & 112 & 68 & 21 & 13 & 17 & 10 & 7 & 4 & 5 & 3 & 4 & 2 & 166 & 100 \\
\hline
\end{tabular}

Fuente: Elaboración personal 


\section{DISCUSIÓN Y COMENTARIOS}

En el estudio de Barrientos Zaidetth y Umaña Roy. «Deserción estudiantil en posgrados semipresenciales de la Universidad Estatal a Distancia (UNED), Costa Rica: ¿Deserción o Retraso? - 2009», se concluye:

[...] que la deserción de los estudiantes se relaciona negativamente con la opinión que tienen sobre el grado de concordancia entre el perfil de salida profesional y las necesidades del mercado laboral, la opinión que tienen sobre la capacidad docente de los profesores y la percepción que tiene el estudiante respecto al nivel de relación entre el trabajo que desempeña el estudiante y la maestría. (Barrientos, Zaidett y Umaña, Roy, 2009).

Estos tres aspectos son de índole personal y están fuertemente relacionados con los aspectos motivacionales que en la presente investigación, también, constituye uno de los factores personales más relevantes asociados al retraso en los estudios y graduación de los estudiantes. Se debe tomar en cuenta la posición crítica que asumen los estudiantes respecto de la organización y calidad educativa de los programas de posgrado, la percepción que estos tengan respecto a la coherencia y calidad de los programas es un componente fundamental para la continuidad y culminación de sus estudios y graduación correspondiente.

Sánchez, Rosalba, en su estudio denominado «El proceso de graduación en el posgrado de pedagogía de la UNAM: el caso de la Maestría de Pedagogía - 2006», encontró que: «el índice de formación y graduación de los estudiantes de posgrado de pedagogía, pueden verse afectados: por la tutoría durante la tesis, falta de prácticas de investigación, falta de interés de los alumnos, entre otros» (Sánchez D., 2011). Estos resultados son, en parte, congruentes con los hallados en la presente investigación, ya que entre los factores institucionales que afectan el retraso en la graduación de los estudiantes se encuentran: la demora, oposición, contradicción y dilatación en la revisión de los proyectos de investigación por parte de los asesores y dictaminadores de tesis (19\%). Este aspecto constituye uno de los factores institucionales más críticos para garantizar la continuidad de las investigaciones y lograr la graduación de los estudiantes, de hecho, una parte importante de los estudiantes que se retrasan en el proceso de graduación lo hacen después de lograr la aprobación de su proyecto de investigación. 
Las conclusiones de Abensur Salomón, en su estudio denominado «Factores socioeconómicos y personales relacionados con la deserción estudiantil en la Escuela de Negocios Internacionales de la Facultad de Ciencias Económicas y Negocios de la Universidad Nacional de la Amazonía peruana, 2002-2006, Loreto - 2009», son confirmadas por la presente investigación, pues efectivamente, son los factores personales (que Abensur califica como factores socioeconómicos y personales) los que están asociados principalmente, al retraso en los estudios y graduación de los estudiantes de posgrado.

En el trabajo de investigación de Deysi, Flores, 2013, «Influencia de los factores Socioacadémicos de los egresados en el proceso de graduación de doctor en la Escuela de Postgrado de la UCSM, 2006-2011», llegó a las siguientes conclusiones: egresados que aún no se han graduado, el $44 \%$ tiene proyecto de tesis, $26 \%$ no tiene proyecto de tesis y solo el $4 \%$ cuenta con borrador de tesis; de los egresados del área ciencias jurídicas y empresariales, ciencias sociales y ciencias de la salud, consideran que los factores socio-académicos que influyen en la no obtención del grado de doctor son, principalmente, condiciones relacionadas con la preparación de los estudiantes, al no tener los conocimientos adecuados que permitan realizar un trabajo de investigación, así como su interpretación y propuesta de medidas de mejora al problema identificado. Los cuales son congruentes con nuestro trabajo de investigación, donde el retraso de graduación coincide en su mayoría con nuestros datos obtenidos: con proyecto representa el $45 \%$, sin proyecto de tesis $24 \%$; redacción del informe $7 \%$; y, con toma de datos de la investigación solo un $2 \%$; lo que refleja que la mayoría de los egresados pueden y tienen la capacidad de realizar el proyecto de investigación, pero en el proceso de ejecutar estos últimos ocurren o se presentaron factores que desalientan y desmotivan a los estudiantes.

En cuanto a los factores que se asocian al retraso de graduación, la realización de la tesis (41\%), fue un factor institucional fuertemente asociado, donde el asesor demoró el avance y le posponían su revisión de tesis; asimismo, el aspecto motivacional (59\%), el factor personal fue asociado al retraso de su graduación, los estudiantes indicaron que dejaron de lado el posgrado ya que este no respondió a sus expectativas y que la tesis fue difícil. Lo que demuestra que la institución educativa debería hacer un seguimiento continuo del estudiante en el proceso de graduación. 


\section{CONCLUSIONES}

PRIMERA. El retraso de estudios en estudiantes de maestrías se presenta mayoritariamente durante el 2.do y 3.er ciclos de estudios. Mientras que para el doctorado no se asoció al retraso de estudios.

SEGUNDA. El retraso de graduación en estudiantes se presentó en su mayoría para maestría y para doctorado, durante la fase de proyecto de investigación.

TERCERA. En cuanto a los factores asociados al retraso de estudios y de graduación, se encuentran los factores institucionales y los factores personales. Los factores personales como la motivación, el aspecto laboral y el económico fueron determinantes, así mismo, dentro de los factores institucionales la coordinación administrativa y la realización de la tesis se encuentran asociadas mayoritariamente.

La hipótesis se comprobó en forma parcial, ya que no solo fueron los factores personales, sino también los factores institucionales los que se encuentran asociados al retraso de estudios y de graduación de los estudiantes de maestría y doctorado.

\section{SUGERENCIAS}

1. La 'comisión de admisión' deberá realizar en sus postulantes una selección mediante la entrevista para verificar su compromiso y seriedad por realizar los estudios de posgrado.

2. El personal administrativo deberá informar y entregar en forma escrita a los estudiantes reglamentos, horarios, requisitos de graduación, entre otros, para ser consultados durante y después de los estudios y puedan realizar sus trámites oportunamente.

3. Los asesores de investigación científica deberán ser una guía en el proceso educativo, informando y aclarando dudas de sus asesorados, mediante una metodología dinámica, práctica y oportuna, acompañando y apoyando a sus asesorados, motivándolos a concluir los trabajos de investigación.

4. Las universidades deben desarrollar mayor investigación, tomando como antecedente el presente trabajo de diagnóstico para llegar a un plan de intervención eficiente y a largo plazo.

Cusco, mayo de 2017 


\section{BIBLIOGRAFÍA}

Barrientos, Zaidett y Umaña, Roy. (21 de setiembre de 2009). Deserción estudiantil en posgrados semipresenciales de la Universidad Estatal a Distancia (UNED), Costa Rica: ¿Deserción o retraso? Obtenido de http://repositorio.uned.ac.cr/reuned/bitstream/120809/1349/1/Barrientos_Umana_Dese rcion.pdf.

Sánchez D., R. (2011). El proceso de graduación en el posgrado de pedagogía de la UNAM: El caso de la Maestría en Pedagogía. Editorial Académica Española. Obtenido de http://redie.uabc.mx/redie/article/view/220/765. 